\title{
Ongoing measles outbreak in Greece related to the recent European-wide epidemic
}

\section{Original Paper}

Cite this article: Georgakopoulou $\mathrm{T}$ et al (2018). Ongoing measles outbreak in Greece related to the recent European-wide epidemic. Epidemiology and Infection 146, 1692-1698. https://doi.org/10.1017/ S0950268818002170

Received: 7 March 2018

Revised: 5 July 2018

Accepted: 9 July 2018

First published online: 8 August 2018

Key words:

Epidemiology; Greece; measles; outbreak

Author for correspondence:

T. Georgakopoulou, E-mail: georgakopoulou. theano@gmail.com

\author{
T. Georgakopoulou ${ }^{1}$, E. Horefti ${ }^{2}$, A. Vernardaki ${ }^{1}$, V. Pogka², \\ K. Gkolfinopoulou ${ }^{1}$, E. Triantafyllou ${ }^{1}$, S. Tsiodras ${ }^{1}$, M. Theodoridou ${ }^{3}$, \\ A. Mentis ${ }^{2}$ and T. Panagiotopoulos ${ }^{1,4}$
}

\begin{abstract}
${ }^{1}$ Department of Epidemiological Surveillance and Intervention, Hellenic Centre for Disease Control and Prevention, Athens, Greece; ${ }^{2}$ National Measles Reference Laboratory, Hellenic Pasteur Institute, Athens, Greece; ${ }^{3}$ National Verification Committee for Measles and Rubella, Athens, Greece and ${ }^{4}$ National School of Public Health, Athens, Greece
\end{abstract}

\begin{abstract}
We report an ongoing measles outbreak in Greece. The first cases were notified through the mandatory notification system on May 2017 in Northern Greece and concerned a cluster of three imported cases in unvaccinated Roma siblings. So far, 3150 cases have been reported in all 13 Regions. Initially, the outbreak mainly affected Greek Roma but as it progressed it spread to non-minority Greek nationals. The outbreak reached its ultimate peak on week $10 / 2018$ but from week 15/2018 has been gradually declining. Most cases (60.6\%) were Roma (especially children $<10$ year-old) followed by non-minority Greek nationals $(29.3 \%$; mostly young adults). The majority (80.4\%) were unvaccinated. Interestingly, 129 (4.1\%) cases were healthcare workers (HCWs). Genotype B3 was identified by molecular methods in all 87 cases tested. Overall, $61.3 \%$ of the cases were hospitalised. Complications were reported in $17.1 \%$ of the cases among which four deaths. The outbreak occurred after 3 years without local endemic measles transmission. Extensive vaccination implemented as the major public health measure managed to prevent the emergence of a large number of cases in refugee/migrant hosting sites. Mitigation efforts currently focus on raising awareness among HCWs and closing the immunisation gap in populations with suboptimal vaccination coverage.
\end{abstract}

\section{Introduction}

Measles is a highly contagious infectious disease which can be eliminated by immunisation program strategies guided by laboratory-supported surveillance.

Measles is a notifiable disease in Greece and surveillance is performed through the mandatory notification system using the European Commission's case definition adapted in 2012 [1].

Both measles and rubella have been targeted for elimination in Greece by 2020, as in the whole WHO European Region, following the WHO Global Measles and Rubella strategic plan 2012-2020 [2]. Throughout the last three decades, the incidence of measles in Greece has presented a constant decline with sporadic clusters or outbreaks of the disease. The last outbreak occurred in 2010-2011 [3]. After 2011 only sporadic measles cases were notified in Greece and as a consequence, according to the European Regional Verification Commission for Measles and Rubella elimination, endemic transmission remained interrupted for a period of 36 months in 2014-2016. Thus, Greece was declared to have achieved elimination $[4,5]$.

Herein, the characteristics of the current measles outbreak in Greece are described. The first cases were notified in May 2017 in Northern Greece. Since then, the outbreak has expanded to various parts of Greece and an increasing number of measles cases have been reported to the Hellenic Centre for Disease Control and Prevention (HCDCP). This led to the implementation of a series of control measures. We report the results of the surveillance and outbreak investigation data analysis as well as the emergency response measures taken.

\section{Methods}

Surveillance data of cases with disease onset from May 2017 to June 2018 were analysed to determine the main characteristics of the outbreak. Medical practitioners and medical laboratories are required to report suspected measles cases, through the mandatory notification system, to the Department of Epidemiological Surveillance and Intervention of the HCDCP. According to the European Commission's case definition, a possible case is defined as any person presenting with fever, maculopapular rash and at least one of cough, coryza, or conjunctivitis (clinical criteria); a probable case is any person meeting the clinical criteria and with an 
epidemiological link to a laboratory-confirmed case; a confirmed case is any person meeting the clinical criteria coupled with the positive measles IgM antibody test or measles virus (MV) isolation result [1]. Following the WHO criteria [6], cases are discarded when clinical, epidemiological or laboratory criteria are not met. Case-based measles notification forms submitted to HCDCP included data concerning the date of disease onset, date of birth and/or age, gender, diagnosis confirmation, vaccination status, hospitalisation, complications and outcome.

Consistent with surveillance guidelines, the incubation period of measles was defined as 7-21 days before rash onset date. Hospital-exposed cases were defined as those who visited any hospital at least once within the incubation period, regardless of the reason for the visit.

The measles vaccination history for each case was recorded and was categorised into four groups: zero doses (unvaccinated), one dose (partly vaccinated), two doses (fully vaccinated), unknown vaccination status. Information on vaccination status was mainly obtained from patient immunisation health records and vaccination booklets, considered to be the best possible source, or in some cases, mostly in the Roma subpopulation group, by self-reporting by the patients. In the latter case, the accuracy of the data could not be checked.

Laboratory confirmation of cases was carried out mainly by the WHO-accredited National Reference Laboratory for measles and rubella - Hellenic Pasteur Institute and/or by nationally accredited laboratories of regional hospitals having an established quality assurance programme (EN ISO 15189). Serum and/or pharyngeal swab samples were collected from the patients. Sera were tested for the presence of IgM antibodies and pharyngeal swabs were tested for the presence of MV RNA with Real-time polymerase chain reaction (RT-PCR).

For measles genotyping, a number of positive samples from laboratory-confirmed cases, representative of the temporal and spatial distribution of measles cases in Greece, were further processed by an RT-PCR amplification of a 598 bp fragment containing the 450 bp hypervariable region of the MV N gene. Genotype assignment was performed by phylogenetic comparison with the MV reference strains as designated by WHO [7].

\section{Results}

Epidemiological findings, genotypic and vaccination coverage data

The first notified measles cases with symptoms onset on 17 May 2017 (week 19) was a cluster of three imported cases in Northern Greece and concerned unvaccinated Roma siblings (6 months, 1 and 6-year-old) of Romanian nationality. From mid-July 2017 up to 17 June 2018 (week 24/2018), the outbreak has spread all over Greece, with 3278 cases reported through the mandatory notification system to HCDCP. It is worth noting that $128 / 3278$ (3.9\%) cases were eventually discarded as non-measles cases by further investigation and negative laboratory testing. From the remaining 3150 measles cases (rate 292.5 cases per 1000000 population) 1839 cases (58.4\%) were laboratory confirmed, 1103 (35.0\%) were classified as probable cases and 206 (6.6\%) were classified as possible.

Genotyping was carried out in 87 cases throughout the investigated period. Sequence analysis revealed B3 as the only measles genotype circulating in Greece. Comparison with sequences deposited in MeaNS database and phylogenetic analysis indicated that the Greek viruses were divided into two main groups (Fig. 1) [7]. Group 1 strains were identical to a recent isolate from Spain, MVs/Badajoz.ESP/18.17/, while those of Group 2 were identical to a recent isolate from Slovenia, MVs/Ljubljana.SVN/27.17. Group 1 viruses were detected at the beginning of the outbreak (week 19/2017) in Northern Greece and subsequently in Central Greece throughout the notification period. The majority of strains tested belonged to Group 2, which was first identified in week 29/ 2017 and has been circulating since then in Central as well as in Southern Greece.

The epidemic curve (Fig. 2) shows the distribution of measles cases per week of symptom onset and population group over time. The number of cases increased rapidly to peaks at week 48/2017 (27 November-3 December) and week 10/2018 (29 February- 4 March). The outbreak is still ongoing at the time of writing this report.

The current outbreak has affected all 13 Greek Regions (according to the Nomenclature of Territorial Units for Statistics - NUTS-2: Attiki, North Aegean, South Aegean, Crete, Eastern Macedonia and Thrace, Central Macedonia, Western Macedonia, Epirus, Thessaly, Ionian Islands, Western Greece, Central Greece, Peloponnese) but mainly those of southern Greece. The highest incidence (1016.61/1000 000 population) was recorded in the Western Greece region, followed by the Peloponnese region (433.37/1 000000 population) while the highest number of cases $(n=866)$ was reported in Attiki region (Fig. 3).

Almost two-thirds of reported cases (1910/3150; 60.6\%) belonged to the Roma population, whilst 924 cases $(29.3 \%)$ belonged to non-minority Greek nationals and 316 (10.1\%) to foreign nationals (Table 1). Out of 22 reported measles cases from Points of Care for refugees/migrants residing in hosting centres, set up after the recent migrant crisis, 17 were laboratory confirmed.

Most cases $(2032 ; 64.5 \%)$ reported so far were children aged 0-14 years. The median age of affected cases was 8 years (range:1 day - 78 years) and 1591 (50.5\%) of the cases were female. The largest number of reported cases $(800 ; 25.4 \%)$ were children aged 1-4 years, while the highest incidence (2916.98/1 000000 population) was recorded in infants below the age of 1 year $(350 ; 11.1 \%)$ (Table 1$)$.

Among the Roma cases, the majority (1675; 87.7\%) were children below 15 years of age and primarily children aged $0-9$ years (1394; 73\%). A total of 235 cases $(12.3 \%)$ occurred in children younger than 1 year of age. More than half of the cases from the non-minority Greek population were young adults aged $25-44$ years $(509 ; 55.1 \%)$ (Table 1$)$.

In total $129(4.1 \%)$ cases occurred among healthcare workers (HCWs), defined as any hospital or other healthcare staff in regular contact with patients. Of the $104 \mathrm{HCW}$ with regular patient contact, 47 were doctors, 39 nurses, four midwives, one biologist, two rescuers and 11 were paramedical personnel. In addition, 25 cases have been reported as supportive personnel at the hospital settings, such as cleaning staff, personnel in the canteen, hospital room assistants, administrative personnel and hospital security personnel. The majority of cases $(64 ; 46.6 \%)$ in HCWs were reported from Attiki region. The median age of affected HCW cases was 39 years (range: 20-56 years). Of the 87 (83.6\%) HCW measles cases with known vaccination status, 39 cases (44.8\%) were reported as partially vaccinated with one dose of MCV while 48 cases (55.2\%) were unvaccinated.

Information concerning vaccination status was available for 2872 measles cases (91.2\%), of which 2534 (88.3\%) were 


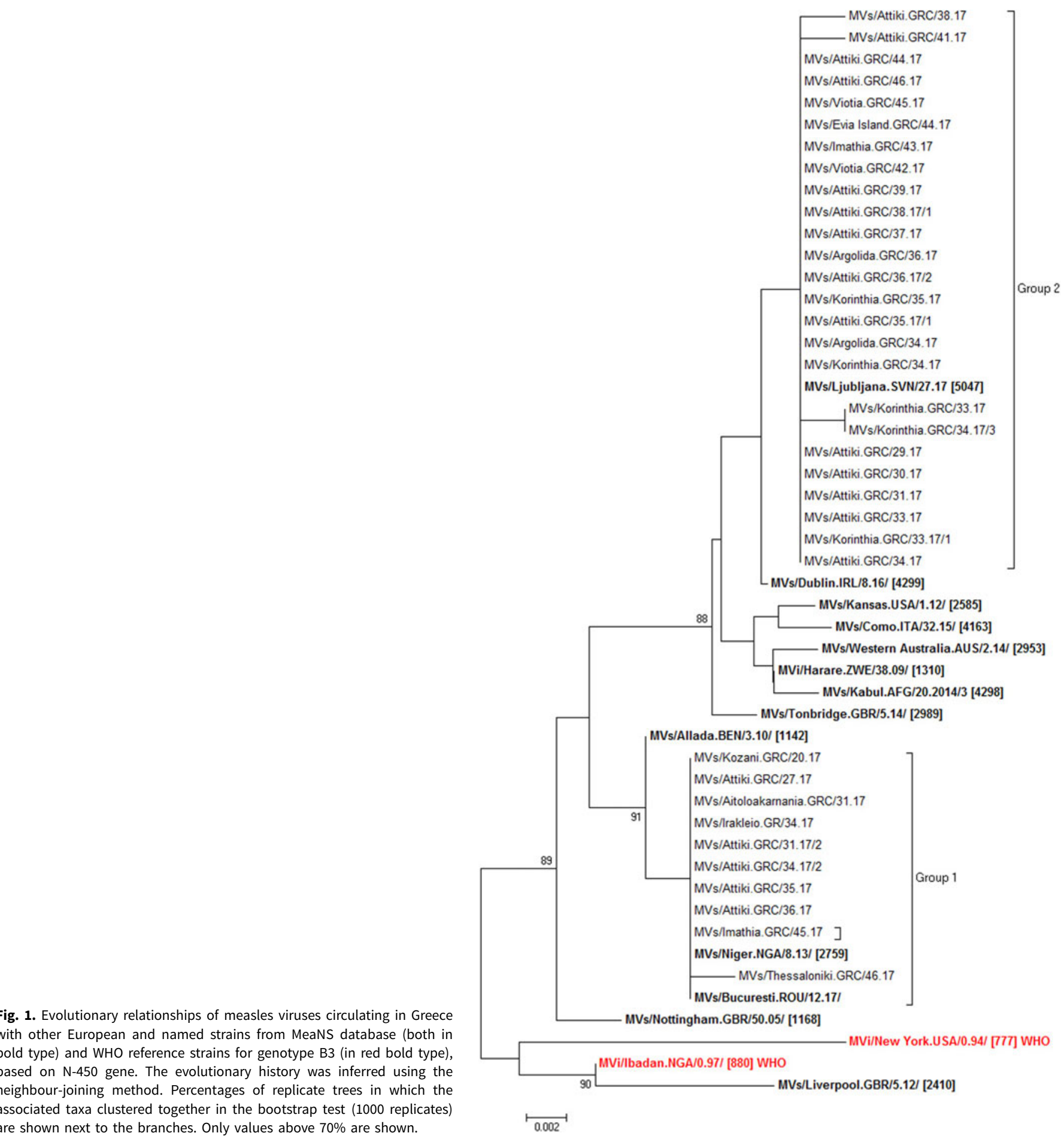

unvaccinated, $42(1.5 \%)$ were vaccinated with an unknown number of doses, 259 (9.0\%) were partially vaccinated with one dose of measles-containing vaccine (MCV) and 37 (1.2\%) with two doses. For the latter cases, vaccine failure was considered as the most probable reason and a report was filed with the Pharmacovigilance office of the National Organization for Medicines. Among the Roma cases, 94.4\% (1741/1844) were unvaccinated and only $5.6 \%$ had received one dose of MCV. The vaccination status of the measles cases of non-minority Greek population was available for 763 cases $(82.6 \%)$. The majority $(554 / 763 ; 72.6 \%)$ of this population was unvaccinated.

\section{Clinical characteristics}

Cases did not always present the classic triad of symptoms following the EU case definition [1]. Some cases were initially not suspected to be measles since symptoms at first presentation included either respiratory symptoms or high fever associated with measles-related complications such as pneumonitis.

In total, $1930(61.3 \%)$ cases were hospitalised. Among 38 cases in pregnant women, $34(89.5 \%)$ were laboratory-confirmed while $31 / 38(81.6 \%)$ were hospitalised. Six pregnant women had pulmonary complications, two had the premature end of pregnancy, 


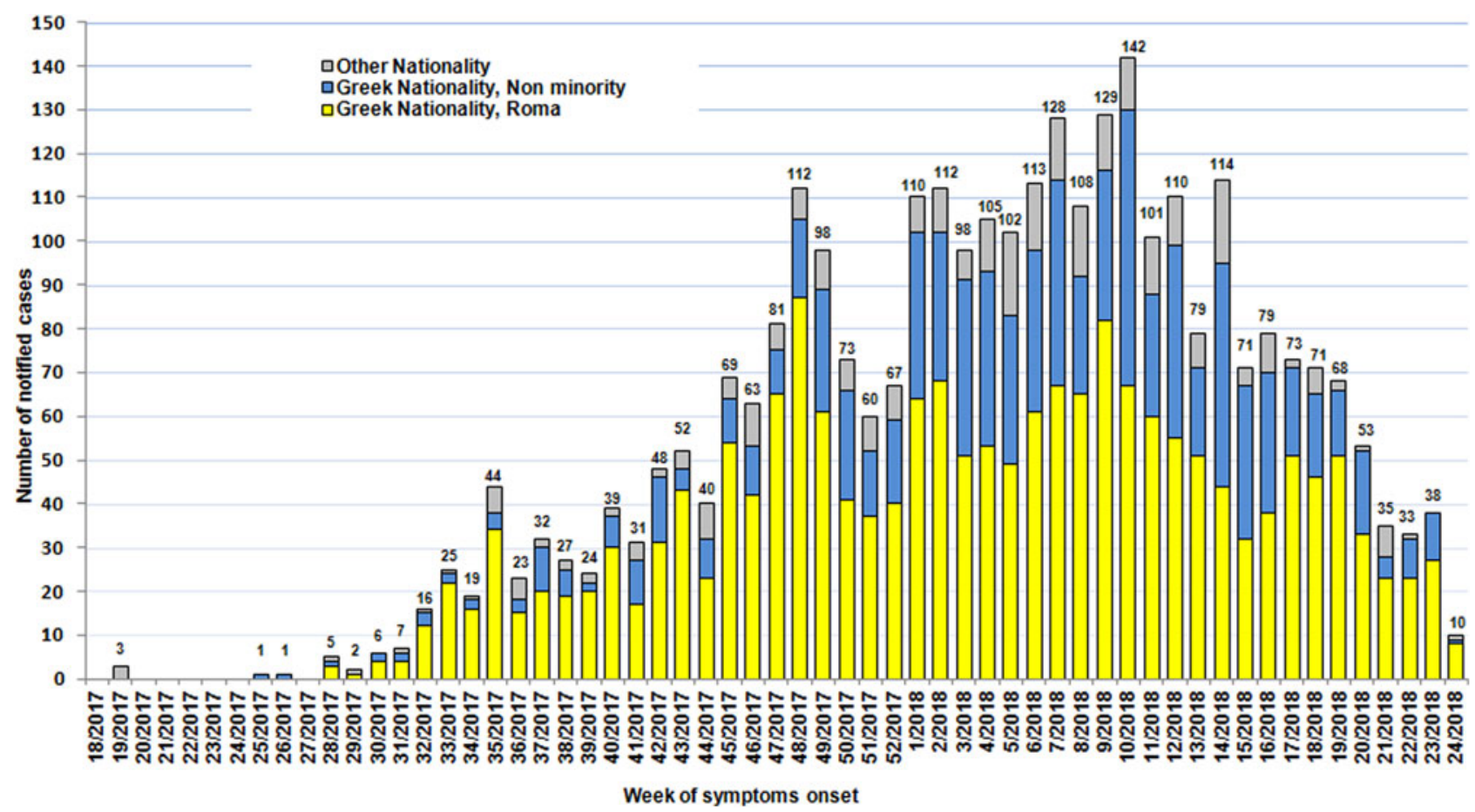

Fig. 2. Reported measles cases per week of symptoms' onset and population group, Greece May 2017-17 June $2018(n=3150)$.
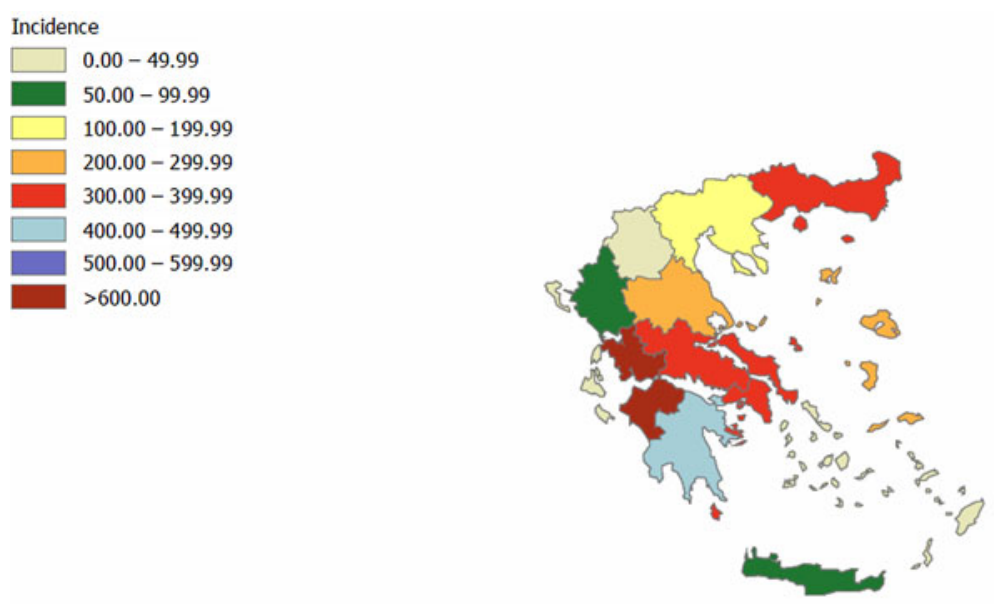

Map produced on: 29 Jun 2018. Administrative boundaries: ${ }^{\oplus}$ EuroGeographics, ${ }^{\odot}$ UN-FAO

Fig. 3. Incidence per 1000000 population of reported cases by Region, Greece, May 2017-17 June $2018(n=3150)$.

one had preterm delivery, one had hepatitis and one had otitis media. In some cases fulfilling the criteria for acute lung injury, IVIG was prescribed at the discretion of the treating physician.

Complications were reported in $540(17.1 \%)$ cases. Among the most frequent complications recorded was pneumonia/pneumonitis $(226 ; 41.8 \%)$, followed by hepatitis manifested by elevated transaminases $(123 ; 22.7 \%)$, otitis media $(87 ; 16.1 \%)$, mild bronchitis/upper respiratory tract involvement $(62,11.5 \%)$, respiratory insufficiency/ARDS $(18 ; 3.3 \%)$, keratitis $(9 ; 1.7 \%)$ and acute encephalitis based on clinician report $(4,0.7 \%)$. The encephalitis case definition developed by the consensus statement of the International Encephalitis Consortium was used [8].

Four deaths were reported in laboratory-confirmed measles cases. The first case concerned an 11-month-old unvaccinated infant, with underlying dystrophy, who died of septicaemia and no other identifiable pathogen in microbiological testing. The second case concerned a 17-year-old unvaccinated adolescent, who died of encephalitis. The third case was a 35-year-old female, 
Table 1. Reported number, percentage and incidence per 1000000 age specific population of reported measles cases by age group in different population groups, Greece, May 2017-17 June $2018(n=3150)$

\begin{tabular}{|c|c|c|c|c|c|c|c|c|c|}
\hline \multirow[b]{2}{*}{ Age group (years) } & \multicolumn{2}{|c|}{$\begin{array}{c}\text { Greek population } \\
\text { Roma }\end{array}$} & \multicolumn{2}{|c|}{$\begin{array}{c}\text { Greek } \\
\text { population } \\
\text { Non-minority }\end{array}$} & \multicolumn{2}{|c|}{$\begin{array}{c}\text { Foreign } \\
\text { Nationality }\end{array}$} & \multicolumn{2}{|c|}{ Total } & \multirow[b]{2}{*}{ Incidence (per 1000000 population) } \\
\hline & $n$ & $\%$ & $n$ & $\%$ & $n$ & $\%$ & $n$ & $\%$ & \\
\hline$<1$ & 235 & 12.3 & 60 & 6.5 & 55 & 17.4 & 350 & 11.1 & 2916.98 \\
\hline $1-4$ & 641 & 33.6 & 76 & 8.2 & 83 & 26.3 & 800 & 25.4 & 2222.46 \\
\hline $5-9$ & 518 & 27.1 & 30 & 3.2 & 25 & 7.9 & 573 & 18.2 & 1043.27 \\
\hline $10-14$ & 281 & 14.7 & 22 & 2.4 & 6 & 1.9 & 309 & 9.8 & 585.69 \\
\hline $15-24$ & 196 & 10.3 & 90 & 9.7 & 26 & 8.2 & 312 & 9.9 & 285.49 \\
\hline $25-34$ & 37 & 1.9 & 210 & 22.7 & 59 & 18.7 & 306 & 9.7 & 235.06 \\
\hline $35-44$ & 2 & 0.1 & 299 & 32.4 & 36 & 11.4 & 337 & 10.7 & 208.32 \\
\hline $45-64$ & 0 & 0 & 132 & 14.3 & 26 & 8.2 & 158 & 5.0 & 54.08 \\
\hline$>65$ & 0 & 0 & 5 & 0.5 & 0 & 0 & 5 & 0.2 & 2.18 \\
\hline Total & 1910 & 100.0 & 924 & 100.0 & 316 & 100.0 & 1784 & 100.0 & 165.67 \\
\hline
\end{tabular}

from the general population, reported as partially vaccinated, who died of pneumonia/Acute Respiratory Distress Syndrome (ARDS) complicated by pulmonary embolism. The fourth case concerned a 18-year-old adolescent, from the general population, with underlying immunodeficiency, fully vaccinated, who died of pneumonia/ARDS. The outcome in all other cases was a cure.

\section{Public health measures}

Following the increasing number of measles cases reported in several European countries in early 2017, the Ministry of Health in cooperation with HCDCP has implemented several public health measures in response to the outbreak. Starting in April 2017, all regional and local public health authorities, committees for nosocomial infections, physicians and other HCWs in the public and private sector were informed about the ongoing outbreak in European countries (especially Romania and Italy) along with appropriate actions regarding isolation of cases, contact tracing and vaccination of susceptible contacts, laboratory investigation and management of affected cases and especially pregnant women and neonates, infection control measures, HCWs vaccination etc. Post-exposure vaccination with MMR within $72 \mathrm{~h}$ to all vulnerable non-pregnant, non-immunocompromised contacts was provided.

HCDCP raised public disease awareness through the distribution of informative material (available on the website of HCDCP; www.keelpno.gr) to all regional public health authorities, hospitals, healthcare centres, medical associations, pharmaceutical associations of Attiki and Piraeus, airports and port authorities, emphasising the importance of immunisation and prevention measures [9]. Key documents concerning measles clinical manifestations, guidelines and background materials for HCWs to support prevention and control measures are available at a dedicated section on measles at the HCDCP website. In addition, since August 2017, HCDCP issues and publishes a weekly and monthly measles report, both in Greek and in English, in the HCDCP's website to provide timely information about the current measles situation in the Regions [9].
In view of the current measles outbreak, a circular was issued by the Ministry of Health/General Directorate of Public Health, where the National Immunization Committee recommended [10]: (a) immediate vaccination of children, adolescents and adults who had not been previously vaccinated with the appropriate doses of the measles-mumps-rubella vaccine (MMR vaccine). According to the National Immunization Program, children, adolescents and adults born after 1970 with no evidence of immunity should receive two doses of MCV, (b) the first dose of MMR to be administered at the age of 12 months and the second dose 3 months later or, if that period has elapsed, as soon as possible. In high-risk cases, the second dose was recommended to be administered at least 4 weeks after the first dose.

Since then, vaccination campaigns in vulnerable population groups with low vaccination coverage have been implemented throughout the country, starting vaccination at the age of 9 months, with priority given to the vaccination of high-risk communities (such as refugee/migrants and Roma).

\section{Discussion}

Measles continues to spread across Europe, causing severe complications and deaths [11]. Clusters of cases have been reported from several countries in Europe in 2016, 2017 and 2018 [12-17]. This is an initial report of an ongoing measles outbreak in Greece, based on notification data obtained from the national surveillance system. The first cluster of cases was among Roma children of Romanian nationality reported in May 2017 and most probably related to the measles outbreak ongoing in Romania since February 2016 [11].

The present measles outbreak started slowly with a few cases in May and June, increasing from mid-July and rapidly progressing from September 2017 onwards and peaking at week 10/2018. The outbreak has mainly affected two groups of the Greek population. The majority of patients were unvaccinated Roma children aged 0-9 years, primarily of preschool age. The 'pre-vaccination era' pattern of the age distribution of measles, in our Greek-Roma communities, underscores the importance of long-term and sustainable vaccination policies for this group. The second most 
affected group was young adults from the non-minority general population mostly between 25 and 44 years of age, who were mainly unvaccinated or partially vaccinated, which underlines the immunity gap in older cohorts. This finding is consistent with reported measles cases in Italy, where adults were the most affected age group, with $68 \%$ of reported cases aged above 20 years [16-17]. In addition, a number of measles cases in fully vaccinated individuals were also reported in Greece, as well as in outbreaks in other countries [18-19] which might be attributed to vaccine failure.

Measles vaccination was introduced in Greece in the early 1970s when vaccines became commercially available and vaccination at the age of 15 months was introduced in the National Immunisation Schedule (NIS) in 1981 with a monovalent vaccine. MMR was first introduced in 1989 and a second dose at the age of 11-12 years was included in the NIS in 1991; in 1999 this dose was shifted to 4-6 years [20]. The last NIS before the recent circular, recommended MMR vaccination at the age of 12-15 months and the second dose at 4-6 years. All vaccines included in the NIS are provided to Greek people free of charge. Since September 2017, as part of the response to this outbreak, MMR immunisation for children belonging to the non-minority general population starts at 12 months of age and the second dose can be administered as soon as 3 months after the first dose, according to established guidance for response to measles outbreaks [10]. Initial immunisation in a vulnerable population (Roma, refugees/migrants) started earlier at the age of 9 months, as a control measure to limit the outbreak as was implemented in Romania [21].

High immunisation coverage with MMR has been recorded in non-minority children in Greece but suboptimal in adolescents and young adults and low in Greek Roma children. According to the Greek national study on vaccination coverage conducted in $2012,98.9 \%$ of first-grade school children (6-years-old) were immunised with one dose of MCV but just $83.3 \%$ with two doses. According to the same study, only $8 \%$ of Greek Roma children had received two doses of MCV [22]. In addition, in the most recent nationwide study on vaccination coverage of children from nurseries to kindergartens conducted in $2014,97.3 \%$ of preschool children aged 2-3 years were immunised with one dose of MCV [23]. Although vaccination coverage with the first dose exceeds $95 \%$, coverage with the second dose is below $90 \%$, lower than the WHO target of $95 \%$ that is necessary for herd immunity [2]. Incomplete vaccination with the second dose of the vaccine might explain the propagation of the epidemic in our country in older ages amongst native non-minority Greeks.

Due to the fact that vaccination against measles has become an essential part of the childhood immunisation schedule, measles has become rare and a large part of the general population, as well as some physicians, seem to have forgotten measles. Therefore, we are confronted with the question of how to effectively raise awareness of the disease and its potential severity and deadly outcome. At the same time, the current outbreak highlights the need to achieve high vaccination coverage with two doses of MMR vaccine in the general Greek population, children as well as adolescents and young adults and the need to increase vaccination in hard-to-reach vulnerable populations such as the Roma population.

Although it is difficult to assess the effectiveness of the updated vaccination policy, we are confident that the implementation of earlier vaccination against measles by HCPs and its adoption by the general public has contributed to the containment of the outbreak. Extensive vaccination with MMR of Roma children as well as children residing in refugee/migrant hosting sites has been undertaken since May 2016 by health services and NGOs with the coordination of the Ministry of Health and HCDCP and has increased further as the outbreak progressed. Nonetheless, vaccination of the Roma population remains a constant challenge and is far from optimal despite all the efforts. Sustained health care policies ensuring good access to vaccination services for the Roma communities as well as tailor-made strategies to reach all susceptible groups are required to close the existing immunity gaps. An important hidden success of our refugee vaccination project which started before the propagation of the epidemic in our country is the lack of cases in this population so far. However, as incoming migrants are continually accepted in hosting centres in Greece, constant awareness is necessary for rapid response and sustaining vaccination efforts are of paramount importance in establishing low measles incidence in this population.

Viruses of Group 1 were first detected in May and circulated in Northern and Central Greece while viruses of Group 2 were first identified in mid-July and circulated in Central and Southern Greece. This spatiotemporal difference in identification of Group 1 and 2 variants may reflect, at least, two different importation events. Further information on different variants circulating around Europe is needed in order to clarify possible chains of transmission and epidemiological links. Genotype B3, which was found in all cases tested, has been circulating during the past year in most European countries, including Italy, Romania, Germany, France [7].

Among the young adults affected in this outbreak, there were 104 HCWs, underlining the fact that hospitals are currently an important transmission setting. By analysing surveillance data, most cases had been hospital-exposed during their incubation periods. Delayed isolation of measles cases in hospital settings led to secondary cases including unvaccinated HCWs. HCWs affected by measles represent a major challenge in containing the epidemic [24-25]. Especially in the cases of HCWs, this is of major concern, since no national legal framework exists to guarantee staff s vaccination against measles; such vaccination is voluntary. Improving two-dose vaccination uptake among HCWs as well as timely implementation of isolation protocols and infection control guidelines in healthcare settings by the committees for nosocomial infections are crucial for the control of the outbreak in Greek hospitals and avoidance of local epidemics in highly vulnerable groups of hospitalised unvaccinated patients e.g. pregnant and immunocompromised individuals.

Some delay between disease onset and diagnosis in the early measles cases could be explained by the fact that physicians might not have considered measles among the diagnoses at the outbreak onset. Gradually, increased awareness among clinicians led to the more timely diagnosis and notification. Similar challenges have impacted control efforts in recent measles outbreaks in other European countries [15, 26].

However, the hospitalisation rate $(61.3 \%)$ was high similarl to other countries experiencing recent outbreaks e.g. Italy and Belgium; furthermore this may possibly reflect under-reporting of mild cases $[11,13]$. High hospitalisation rates may be explained by the fact that clinical awareness about the disease has been low over the last decade and differential diagnosis especially at the early stages of the disease when high fever and cough predominate, is difficult to make from other diseases like the concurrent influenza presence during the winter months. In addition, the 
higher risk of complications in the adults might have led to a high rate of admissions in the current outbreak.

This ongoing outbreak provides the opportunity to further study routes of transmission, to evaluate control measures implemented but also to learn lessons that can be valuable in the National Immunization program's effort towards the goal of measles elimination.

Acknowledgements. Source of data: National Mandatory notification system (HCDCP), National Reference Laboratory for Measles and Rubella Hellenic Pasteur Institute

The authors would like to thank all clinicians reporting measles cases to the national surveillance system, the colleagues from regional public health authorities involved in cases investigation and the members of the National Verification Committee (NVC) for the elimination of measles and rubella in Greece for their support. The authors also like to thank Panagiotis Katsaounos (Department of Epidemiological Surveillance of HCDCP) and Pantelis Mavraganis (Department of Epidemiological Surveillance of HCDCP) for assisting with data management and compilation of information.

Declaration of interest. None.

\section{References}

1. European Commission (2012) Commission Implementing Decision of 8 August 2012 amending Decision 2002/253/EC laying down case definitions for reporting communicable diseases to the Community network under Decision No 2119/98/EC of the European Parliament and of the Council. Luxembourg: Publications Office of the European Union. L262 27 September 2012. Available at http://eur-lex.europa.eu/legal-content/EN/ TXT/?uri=OJ\%3AL\%3A2012\%3A262\%3ATOC (Accessed 5 March 2018).

2. World Health Organization (WHO) (2012) Global Measles and Rubella Strategic Plan 2012-2020. Geneva: WHO. Available at http://apps.who.int/iris/ bitstream/10665/44855/1/9789241503396_eng.pdf (Accessed 5 March 2018).

3. Pervanidou D et al. (2010) Spotlight on measles 2010: ongoing measles outbreak in Greece, January-July 2010. Euro Surveillance 15, pii=19629.

4. World Health Organization Regional Office for Europe (WHO/ Europe) (2017) Fifth meeting of the European Regional Verification Commission for Measles and Rubella Elimination (RVC) 24-26 October 2016, Copenhagen, Denmark. Copenhagen: WHO/Europe. Available at http://www.euro.who.int/en/health-topics/communicable-diseases/measlesand-rubella/publications/2017/5th-meeting-of-the-european-regionalverification-commission-for-measles-and-rubella-elimination-2017 (Accessed 5 March 2018).

5. World Health Organization Regional Office for Europe (WHO/Europe) (2017) Sixth meeting of the European Regional Verification Commission for Measles and Rubella Elimination (RVC) 15-17 June 2017, Bucharest, Romania. Copenhagen: WHO/Europe. Available at http://www.euro.who. int/en/health-topics/communicable-diseases/measles-and-rubella/publications/ 2017/6th-meeting-of-the-regional-verification-commission-for-measlesand-rubella-elimination-rvc) (Accessed 5 March 2018).

6. World Health Organization (WHO) (2014) Eliminating Measles and Rubella - Framework for the Verification Process in the WHO European Region 2014. Copenhagen: WHO. Available at http://www.euro.who.int/ __data/assets/pdf_file/0009/247356/Eliminating-measles-and-rubellaFramework-for-the-verification-process-in-the-WHO-European-Region. pdf) (Accessed 15 June 2018).

7. World Health Organization (WHO) Measles Nucleotide Surveillance (MeaNS) Database. Geneva: WHO. Available at http://www.who-measles. org/Public/Web_Front/clinical.php) (Accessed 5 March 2018).

8. Venkatesan A et al. (2013) Case definitions, diagnostic algorithms, and priorities in encephalitis: consensus statement of the international encephalitis consortium. Clinical Infectious Diseases 57, 1114-1128.

9. Hellenic Centre for Diseases Control and Prevention (HCDCP) Measles outbreak in Europe and in Greece. Available at http://www.keelpno.gr/el-
gr/\%CE\%B1\%CF\% $81 \% C F \% 87 \% C E \% B 9 \% C E \% B A \% C E \% B 7 / m e a s l e s \_h i g h-$ light2017.aspx (Accessed 5 March 2018).

10. Greek Ministry of Health (2017) Measles outbreak - Vaccination Decision of 21 September 2017. Available at https://diavgeia.gov.gr/doc/ \%CE\%A9893465\%CE\%A6\%CE\%A5\%CE\%9F-8\%CE\%98\%CE\%9D?inline=true (Accessed 5 March 2018).

11. European Centre for Disease Prevention and Control (ECDC) (2018) Monthly measles and rubella monitoring report - June 2018, Stockholm. ECDC. Available at https://ecdc.europa.eu/sites/portal/files/ documents/Monthly-Measles-Rubella-monitoring-report-June-2018.pdf (Accessed 28 June 2018).

12. European Centre for Disease Prevention and Control (ECDC) (2017) Rapid Risk Assessment. Ongoing outbreak of measles in Romania, risk of spread and epidemiological situation in EU/EEA countries - 3 March 2017, Stockholm. ECDC. Available at https://ecdc.europa.eu/sites/portal/ files/media/en/publications/Publications/27-02-2017-RRA-Measles-Romania\% 2C\%20European\%20Union\%20countries.pdf (Accessed 5 March 2018).

13. Grammens T et al. (2017) Ongoing measles outbreak in Wallonia, Belgium, December 2016 to March 2017: characteristics and challenges. Euro Surveillance 22, 30524.

14. George F et al. (2017) Measles outbreak after 12 years without endemic transmission, Portugal, February to May 2017. Euro Surveillance 22, 30548.

15. Filia A et al. (2017) Ongoing outbreak with well over 4,000 measles cases in Italy from January to end August 2017 - what is making elimination so difficult? Euro Surveillance 22, pii=30614.

16. European Centre for Disease Prevention and Control (ECDC) (2018) Risk of measles transmission in the EU/EEA - 23 March 2018, Stockholm. ECDC. Available at https://ecdc.europa.eu/sites/portal/files/ documents/Measles-rapid-risk-assessment-European-Union-countries_0. pdf (Accessed 28 June 2018).

17. European Centre for Disease Prevention and Control (ECDC) (2018) Measles and rubella surveillance - 2017, Stockholm. ECDC. Available at https://ecdc.europa.eu/en/publications-data/annual-measles-and-rubellamonitoring-report-2017 (Accessed 28 June 2018).

18. Hahni SJ et al. (2016) Measles outbreak among previously immunized healthcare workers, the Netherlands, 2014. The Journal of Infectious Diseases 214, 1980-1986.

19. Muscat M et al. (2016) The measles outbreak in Bulgaria, 2009-2011: an epidemiological assessment and lessons learnt. Euro Surveillance 21, 30152.

20. Georgakopoulou T et al. (2006) Current measles outbreak in Greece. Euro Surveillance 11, pii=2906.

21. Romanian Ministry of Health (2016) Ministerul Sănătății a luat măsuri suplimentare pentru limitarea epidemiei de rujeolă (The Ministry of Health has taken additional measures to limit the epidemic of measles 16 December 2016) (In Romanian). Available at http://www.ms.ro/2016/ 12/16/ministerul-sanatatii-a-luat-masuri-suplimentare-pentru-limitareaepidemiei-de-rujeola/ (Accessed 5 March 2018).

22. Panagiotopoulos T et al. (2012) Report: Greek national study on vaccination coverage in children, 2012. (In Greek). Available at http://www.nsph. gr/files/011_Ygeias_Paidiou/Ereunes/ekthesi_emvolia_2012.pdf (Accessed 5 March 2018).

23. Georgakopoulou T et al. (2017) A cross-sectional vaccination coverage study in preschool children attending nurseries-kindergartens: implications on economic crisis effect. Human Vaccines \& Immunotherapeutics 13, 190-197.

24. Maltezou HC et al. (2018) Measles in healthcare workers during the ongoing epidemic in Greece, 2017-2018. The Journal of Hospital Infection 11 Jun 2018. pii: S0195-6701(18)30321-9. doi: 10.1016/ j.jhin.2018.06.007. [Epub ahead of print].

25. Maltezou HC and Wicker S (2013) Measles in health-care settings. American Journal of Infection Control 41, 661-663.

26. Barrett P et al. (2016) An ongoing measles outbreak linked to a suspected imported case, Ireland, April to June 2016. Euro Surveillance 21, pii $=30277$. 